Instituto Internacional de Investigación y Desarrollo Tecnológico Educativo INDTEC, C.A.

DOI: https://doi.org/10.29394/scientific.issn.2542-2987.2016.1.2.8.131-146

OAI-PMH: http://www.indteca.com/ojs/index.php/Revista Scientific/oai

\title{
Supervisión Vs Calidad Educativa en Educación Primaria
}

\author{
Autor: Jhoel Ruber Mota Fonseca \\ Universidad Fermín Toro, UFT \\ jhoelmota@hotmail.com \\ San Felipe, Venezuela
}

\begin{abstract}
Resumen
El presente artículo posee como objetivo interpretar en las voces de los docentes la supervisión vs calidad educativa en Educación Primaria, ya que hoy en día, la supervisión supone el progreso y perfeccionamiento en el desarrollo de las funciones del docente en el ambiente de aprendizaje, es por ello, que a través de este contenido se suministra información de los conflictos presentes para alcanzar cambios dentro de las instituciones escolares en pro de la eficiencia y virtud organizacional. En el mismo, se establece un enfoque paradigmático cualitativo como fundamento metodológico que permite interpretar la supervisión educativa a través delos actores sociales el cual está conformado por el personal directivo, docente y supervisores en el contexto de la educación primaria, así mismo fue necesario recoger la información a través de la técnica de observación directa utilizando como instrumento un guion de entrevista cuya aplicación fue a los informantes clave conformado por un docente de educación primaria, un supervisor y entre ellos el investigador, logrando así el análisis correspondiente de acuerdo a la información suministrada por cada uno de ellos, que permitió el desarrollo de competencias argumentativas en los docentes, estableciendo reglas y procedimientos que dan lineamientos para la creación de respuestas al problema planteado. Por con siguiente se aspira determinar en la investigación, los vitales agentes que contribuyan a que los maestros sean altamente competentes en su práctica diaria, utilizando estrategias pedagógicas que le permita el éxito dentro del aula de clase, cuyo propósito es la orientación al logro y la optimización en el proceso de enseñanza-aprendizaje de los estudiantes. En este sentido, se realiza una reflexión en relación al papel que debería desempeñar el supervisor para lograr la calidad educativa, evidenciándose los espacios de mediación, Integración, participación y colaboración con respecto a la actuación profesional del maestro cuyo fin es ser eficiente y eficaz en su labor pedagógica del sistema educativo.
\end{abstract}

Palabras clave: supervisión; calidad educativa; educación primaria.

Fecha de Recepción: 14-08-2016

Fecha de Aceptación: 05-09-2016 


\title{
Supervision Vs Education Quality in Primary Education
}

\begin{abstract}
This article has as objective to interpret the voices of teachers supervising vs quality of education in primary education, as today, supervision entails progress and improvement in the performance of the duties of teachers in the learning environment is therefore, that through this content conflicts present information to achieve changes within educational institutions towards organizational efficiency and under supplied. In it, a qualitative paradigmatic approach as a methodological foundation that allows us to interpret educational supervision through delos social actors which is made up of managers, teachers and supervisors in the context of primary education, established likewise was necessary to collect the information through direct observation technique using as an interview script whose implementation was key informants comprised of a primary teacher education, a supervisor and from this the researcher, thus achieving the corresponding analysis according to the information provided for each, which allowed the development of argumentative skills teachers, establishing rules and procedures that provide guidelines for creating answers to the problem. For with following aims determined in the investigation, vital agents that help teachers to be highly competent in their daily practice, using teaching strategies that will enable success in the classroom, whose purpose is the achievement orientation and optimization in the process of teaching and student learning. In this sense, a reflection about the role it should play the supervisor to achieve educational quality is performed, demonstrating spaces mediation, integration, participation and collaboration with respect to the performance of the master whose aim is to be efficient and effective in its educational work of the educational system.
\end{abstract}

Keywords: supervision; quality education; primary education.

Date Received: 14-08-2016

Date Acceptance: 05-09-2016 


\section{Introducción}

Desde el comienzo de la historia del hombre en el planeta tierra, ha visto la inquietud de evaluar, controlar y reorientar las actividades que él con sus semejantes realizan en un tiempo y espacio determinado. Esta cualidad por supuesto la heredó de Dios, quien al hacerlo a su imagen y semejanza, lo dotó de algunas características de su creador: supervisor por excelencia. El libro de Génesis narra cómo el Ser Supremo en todo momento supervisó y evaluó su maravillosa creación al manifestar que todo lo creado "era muy bueno" (Gen 1:31). Este pasaje bíblico, es sólo uno de los ejemplos de cómo la necesidad de supervisar el trabajo, es algo con lo que Dios ha dotado al hombre para su desenvolvimiento en este planeta, lo cual la historia corrobora desde las primeras civilizaciones que se formaron después del diluvio, como la Egipcia, Asiría, Mesopotámica-babilónica, Medopersa, Griega y Romana.

De allí que, a lo largo de la evolución histórica todas las organizaciones emplean diversos mecanismos para lograr las actividades que desarrollan, ya que cada proceso posee su propia naturaleza y forma de hacerlo; es por ello que utiliza la supervisión, como acción de verificar el cómo y con que lo están realizando en caso específico en la educación, para mejorar el trabajo pedagógico, el cual ha sido elemental para el desarrollo de la humanidad en general.

Se debe tener presente, como las naciones se desenvuelven a partir de la educación de sus ciudadanos, sería lógico pensar que desde tiempos remotos la educación haya estado presente en este apartado solo como instrumento de verificación y perfección de la calidad de la enseñanza, al respecto en palabras de Ortega (2012) la educación se puede comprender como la destinación para ampliar la capacidad que posee cada ser humano apoyado en lo moral y afectivo, partiendo de su cultura y de la normativa que rige la sociedad; por ello se le denomina un bien público y derecho humano 
donde nadie puede estar excluido bajo ningún contexto social con llevándolo así a la libertad por medio del proceso de la educación.

Parafraseando las palabras del autor, el cual refiere que la educación a través de la evolución a permitido al individuo ampliar su capacidad bajo una adecuada moral y afectividad sin dejar a un lado su cultura dentro de su propio contexto en el que se desenvuelva para llegar a su propia libertad dentro de la normativa establecida en la sociedad. En el proceso evolutivo de la educación, ha jugado un papel fundamental en la formación intelectual, artesanal y militar del hombre; donde la mayoría de las veces con una rigidez extrema bajo una disciplina autocrática basada en el castigo que con el paso del tiempo han ido cambiando y logrado grandes avances. A partir de esos desafíos, la educación requiere de una actuación participativa y cooperativa de todos dentro del proceso en la escuela, con la finalidad de tener influencia sobre todas las situaciones de aprendizaje de formación integral en el centro educativo.

Actualmente se busca adecuar el sistema educativo a los procesos de reforma que adelanta el Estado venezolano como respuesta a los nuevos requerimientos políticos, educativo, económicos y sociales en que la educación tiene misión de formar al individuo con el fin de enfrentar las transformaciones en los procesos y consolidar los valores indispensables para una sociedad de avances que exige una concepción distinta a la tradicional permitiendo reorientar la acción educativa a través de la supervisión para obtener una calidad educativa. Es por ello que la supervisión, como componente de la administración educativa, lleva implícita la labor de orientación oportuna, por lo que requiere de un personal especializado, con adecuada formación pedagógica para ofrecer al supervisado estrategias para el proceso de aprendizaje y así afrontar los problemas que se presentan en la práctica.

Este sentido, para generar un cambio en este nivel de la profesión docente, es indispensable incidir en la génesis del sistema educativo a fin de 
redefinir la política normativa y estructura del sistema nacional de supervisión. A través de la revisión de diversas fuentes bibliográficas, me lleva a plantear la siguiente inquietud, ¿Se hace necesario adecuar el modelo de supervisión actual con el sistema educativo para obtener una calidad educativa en la educación primaria? De lo planteado me permite direccionar el propósito general del presente artículo en Interpretar en la voz de los docentes la supervisión educativa vs calidad educativa en la educación primaria

\section{Sistema Educativo}

Hoy en día se conoce que el sistema educativo se ha venido deteriorando paulatinamente, lo que da lugar a que se realicen fuertes críticas donde se cuestiona el papel de la educación en las instituciones escolares. En este sentido, desde un punto de vista sistemático e institucional, quien dispone de políticas y servicios para atender los requerimientos de los problemas presentes en el desarrollo de la práctica pedagógica. Para tal fin, el mismo posee una serie de estructuras administrativas que determinan la existencia de unidades de planificación, organización, dirección y supervisión, orientados hacia el alcance de los objetivos mediante la integración y uso eficiente de recursos económicos, materiales y humanos existentes.

Mediante estos procesos administrativos las organizaciones educativas a través del sistema educativo buscan el perfeccionamiento de su productividad y calidad de bienes y servicios que prestan a la sociedad. Esencialmente el control es la función administrativa en la cual, se fija metas y se busca información sobre si éstas se alcanzaron como verdaderamente se planeó. De no ser así, se deben hacer los ajustes necesarios para asegurarse que se están cumpliendo los objetivos propuestos. La función encargada de ejercer ese control es la supervisión, siendo su principal objetivo producir cambios positivos en los procesos pedagógicos-administrativos en el 
desarrollo del proceso de enseñanza y aprendizaje presentes en el sistema educativo.

De allí que, es pertinente la temática abordada para la investigación porque que la supervisión educativa juega una papel importante en la educación actual porque permite la unidad, corresponsabilidad, interdependencia y flexibilidad en los programas curriculares, garantizando en el docente la formación permanente con el respeto a sus capacidades, a la diversidad, lingüística y cultural cuyo propósito debe ser atender a las necesidades y potencialidades del profesional dentro del sistema educativo.

\subsection{Supervisión}

El proceso de supervisión en el continente latinoamericano y de manera especial en Venezuela se ha visto afectado por una serie de factores tanto internos como externos que no permiten que se desarrolle de manera eficiente. En este sentido, la Organización de las Naciones Unidas para la Educación, Ciencia y Tecnología (UNESCO 1982) expresa que la supervisión en América Latina: carece de principios definidos que la orienten hacia los objetivos y necesidades legítimas de la educación; así mismo existe una disparidad entre lo que se planifica y su ejecución debido que lo primero siempre está por encima de las posibilidades (pág. 15).

Tomando la idea de la UNESCO, puedo inferir que la supervisión presenta debilidad en los principios los cuales guían el logro de las metas planteadas, sin embargo, existe incongruencia en lo planificado con lo ejecutado, es decir se trabaja sin basamento previo establecido lo cual repercute en la ejecución de la misma dentro de una organización escolar. Adicionalmente, es notoria la forma con la cual es tratado el proceso de supervisión, los mismos son diseñados con modelos de países desarrollados, con realidades y factores distintos, no adaptables a nuestro contexto como país. Siguiendo la misma idea, por su parte Marín (2012) expresa que: "la 
supervisión se centraliza en el aula tradicional-impositiva, sin tomar en cuenta la creatividad y las vivencias de los educandos, educadores y demás miembros de la comunidad”. (pág. 87)

De acuerdo, con lo expresado en las citas anteriores es evidente la disociación entre lo que se quiere lograr en materia educativa y lo que realmente se hace, tanto por la carencia de recursos como por la irrealidad de las expectativas planificadas; el desfase entre lo que se planifica y se ejecuta, la supervisión fiscalizadora y la tardanza en la toma de decisiones ocasionada por el excesivo centralismo, entre otros elementos, no permiten elevar la calidad de la educación en la educación primaria.

Realizando una senda por los desempeños habituales de la supervisión, los cuales poseen una connotación muy importante en el quehacer educativo, dándole especial preponderancia a aquellas donde el funcionario a cargo de ella coloca su atención sobre las actividades asignadas, especifica en informar, asesorar, mediar, controlar, evaluar y que engloba bajo la designación de supervisión haciendo sinónimos los términos de: vigilancia, control, acompañamiento, fiscalización, entre otros. De lo referido, se infiere que la supervisión como vía entre la administración educativa y el desempeño docente se asienta en su considerada responsabilidad al adecuado sistema y requerimientos de la sociedad. En lo educativo, se considera el control y la evaluación en sus campos, los cuales permiten garantizar el derecho del niño, niña y adolescentes a una educación participativa, protagónica y de calidad en la educación primaria.

Es preciso denotar, que aún se conservan funciones relacionadas con la actualización permanente del profesorado, pero éstas han desaparecido casi por completo del horizonte, al menos como encargo específico desde la administración educativa lo cual afecta a la supervisión; lo planteado lleva a determinar de forma genérica, que la supervisión se encarga habitualmente, de informar, asesorar, mediar, controlar y evaluar todo el desarrollo del 
proceso educativo que se ejecuta en las escuelas y es por medio de este que se logra palpar la realidad en cuanto al desarrollo pedagógico y adecuada ejecución de las funciones docente.

El enfoque que posee la supervisión, en el sistema educativo de Venezuela, hace que sea la instancia adecuada para ejercer la calidad educativa requerida en los planteles de educación primaria, donde se observa que la supervisión se encuentra entre la administración educativa y el desempeño del docente, quien realiza una labor de puente entre ambos. Es decir; las normativas de la administración educativa llegan a la escuela a través de la supervisión y el funcionamiento obtenido en la aplicación de las normas, exigencias sociales o necesidades de la escuela, también se logran por medio del mismo a la administración educativa. Esta situación convierte la supervisión en clave del desarrollo de los procesos de calidad educativa, pues de su correcto funcionamiento depende la adecuación rápida de los requerimientos de la institución educativa.

Al igual que, es responsabilidad de la supervisión que las innovaciones propuestas desde la administración educativa se apliquen, de forma efectiva, en los centros educativos de educación primaria. Es por ello que, los supervisores a través de la supervisión garantizan el ajuste de la calidad en el sistema educativo con su aplicación rápida y apropiada. Como asegura la tradicional afirmación de Gil de Zárate, (2009), refiriéndose a los supervisores: "Si en todos los ramos del servicio público es conveniente esta clase de funcionarios, en la instrucción pública es indispensable. Sin ellos la Administración nada ve, nada sabe, nada puede remediar". (pág. 31) Basando en la definición del autor, es conveniente que existan estos funcionarios, porque sin ellos no se obtendría información e incluso avance o debilidad en el desempeño de las funciones del docente de aula, las cuales permiten conocer a profundidad lo que se hace, como, el deber ser respectivos, en las instituciones educativas. 
La supervisión en la educación primaria, se hace necesaria que la desarrolle un supervisor con una visión amplia, basado en el humanismo, sentido de pertenencia y pertinencia pero que a su vez lleve a sus docentes hacia el ejercicio eficaz de sus competencias estableciendo el logro de los objetivos institucionales con la finalidad de garantizar los derechos de los estudiantes a una educación de calidad. Aunque, algunas veces, se evidencie muy burocráticas las tareas delegadas del supervisor, ya que no puede perder de vista las metas esenciales de su quehacer. Y, sobre todo, no olvidar: lo urgente no debe relegar lo importante, pues es grande el riesgo para las escuelas de educación primaria.

La garantía de una educación de calidad, para todo el estudiantado, fundamentalmente al que se encuentra en edades de escolarización obligatoria, es responsabilidad directa del supervisor, pues constituye el aval de que se cumple, en la sociedad y escuela, lo que determina la administración educativa. Por otro lado, la estrategia fundamental que dispone la supervisión para que esa garantía sea una realidad, es el ejercicio de su función evaluadora mediante la aplicación de un modelo de apreciación formativa y continúa desarrollado en la escuela, la cual favorezca el ajuste y la innovación permanente de los hechos educativos que en ella se producen a diario, para tener así una educación de calidad

De lo antes mencionado, se puede inferir que la supervisión optimiza el rendimiento del sistema educativo mediante la aplicación de la evaluación formativa, que tiene como objetivo el perfeccionamiento de los procesos educativos (de enseñanza y aprendizaje). Si se mejoran los procesos, se puede llegar a obtener resultados de calidad en pro de nuestros escolares, porque obtendrían una mejor formación gracias a la calidad educativa desarrollada por el docente y guiada por el supervisor en la educación primaria. 
Sin embargo, hay que aceptar que, todavía los supervisores al momento de tratarse de supervisar a la totalidad del sistema y no sólo al estudiante sino al docente, habría que admitir, igualmente, que la supervisión tampoco goza de una buena imagen dentro del sistema. Si se actualizara el modelo de supervisión, se transformaría en un instrumento válido para perfeccionar el quehacer educativo en general para el trabajo dentro de las escuelas, en el modelo de enseñanza, en el aprendizaje del escolar, en la participación activa y positiva de los agentes sociales, en el ejercicio de la dirección, se transformarán en medios aceptados y asumidos como imprescindibles para conseguir la calidad educativa pretendida en la educación primaria.

Se debe recordar que la sociedad evoluciona a ritmos sumamente acelerados, en algunos aspectos que influyen o deberían influir en los planteamientos institucionales de la educación primaria. Tanto por lo que se refiere a la tarea que se desarrolla en el centro y en el aula, como en el diseño estructural de la administración educativa para que los agentes que colocan en marcha cumplan con eficacia las funciones que se requieren en los actuales momentos.

Como en el caso particular, el avance en el campo del conocimiento, la incorporación de la tecnología a todos los ámbitos de la vida sobre todo de la información y la comunicación, la globalización en que derivan los dos puntos anteriores, la movilidad de las personas a nivel mundial. Todo ello, como decía, a una velocidad que casi hace imposible la adaptación individual a las nuevas situaciones. Este nuevo mundo exige un cambio de actitudes que, en efecto, no se logra de un día para otro, pero que nos hace vivir, presencial o virtualmente, en contextos de pluralismo ideológico y cultural cada vez más diversos. Que a su vez obligan a estar a la vanguardia de los cambios y lo más idóneo la educación no escapa de esta realidad, razón por lo cual se debe tener presente que los cambios permiten avanzar y cambiar paradigmas que llevan al éxito de lo que promulgamos calidad educativa. 
De acuerdo al razonamiento que se viene planteado, se infiere su relación con la presente investigación porque la supervisión en contexto educación primaria, representa un proceso esencial para la mejora continua de los centros educativos y del docente. Sin ella no se podría ver si la institución educativa está cumpliendo con el programa o los objetivos establecidos por los administrativos, no tampoco los docentes sabrían si los estudiantes están aprendiendo lo establecido por el currículo. De manera que, la supervisión educativa debe ser vista y utilizada para la mejora continua de los centros educativos y de su personal, permitiendo crear soluciones inmediatas en búsqueda de la calidad educativa.

\subsection{Educación Primaria}

El hecho cierto, es que la educación primaria, se percibe como multicultural y cambiante, a lo que se añaden las diferencias personales de cada individuo en capacidades, situaciones transitorias o formas de entender la vida, aun dentro de un mismo grupo cultural y las que la propia sociedad presenta o ella misma genera entornos marginales, migración, grupos étnicos o culturales minoritarios, desconocimiento de la lengua mayoritaria. El cambio seria la constante de que se dispone, y en base a estas realidades, será necesario establecer modelos de estrategias que resulten funcionales para la calidad educativa de las nuevas generaciones.

La realidad social que se evidencia en las escuelas, significa que en cada una de ellas se deba atender las diferentes necesidades educativas, en función de la población que escolariza en beneficio de la calidad educativa en el que desenvuelve su trabajo. Y si se acepta esta premisa, habrá que reconocer la autonomía de la escuela para que ésta pueda cumplir con las funciones que tiene encomendadas. El mismo planteamiento es válido para la supervisión. No hace tantos años que los supervisores debían controlar para garantizar que todas las escuelas poseían las mismas necesidades. Es decir, 
que en ellas se cumplía el mismo programa, de la misma manera, con horarios similares, con organización idéntica.

Si nos encontramos con la realidad descrita, la supervisión no cambia, ciertamente, pero sí su contenido: ahora el supervisor debe controlar que todas las escuelas sean diferentes; como es fácil deducir, para que sean capaces de atender a su población específica. Los niños presentan una gran diversidad; los contextos y grupos sociales, también por lo tanto, tendrán que ofrecer respuestas adecuadas a esa diversidad de situaciones personales y sociales. Porque la supervisión, controla y evalúa que eso se realice de la manera más idónea. Pero; no cambia la función, pero sí su contenido. De este modo, se garantizará la calidad educativa para toda la población escolar, es decir, unir calidad y equidad, consiguiendo calidad educativa para todos.

De modo que, se puede considerar que el tópico mencionado gualda relación con la presente investigación ya que la educación primaria debe estar enmarcada en los paradigmas actuales de la supervisión educativa, donde permita formar niños y niñas activos, reflexivos, críticos e independientes, con elevado interés para la actividad científica, humanista y artística; con un desarrollo de la comprensión, confrontación y verificación de su realidad por sí mismo y sí misma; con una conciencia que les permita aprender desde el entorno y de ser cada vez más participativos, protagónicos y responsables en su actuación en la escuela, la familia y la comunidad.

\section{Metodología}

En relación a la metodología empleada se establece un enfoque paradigmático como fundamento metodológico que permita interpretar la supervisión educativa a través de los actores sociales el cual está conformado por el personal directivo, docente y supervisores en el contexto de la educación primaria, así mismo fue necesario recoger la información a través de la técnica de observación directa utilizando como instrumento un guion de entrevista 
cuya aplicación fue a los informantes clave conformado por un docente de educación primaria, un supervisor y entre ellos el investigador, logrando así el análisis correspondiente de acuerdo a la información suministrada por cada uno de ellos, que permitió el desarrollo de competencias argumentativas en los docentes, estableciendo reglas y procedimientos que dan lineamientos para la creación de respuestas al problema planteado

La investigación cualitativa, ofrece la oportunidad de percibir la supervisión en su acción diaria en las instituciones educativas a través del sistema educativo en su acción y quehacer se rige por reglamentos legales de los instrumentos jurídicos que administran las políticas educativas del país y que, caracterizan el proceso educativo y el tipo de supervisor que se requiere para intervenir en la educación primaria.

Al respecto Márquez (2010) refiere que, en la investigación cualitativa, el diseño sufre cambios para adaptarse al contexto. Posibilitando el libre flujo de versiones y significantes, para darle sentido, y así proceder a su interpretación. Es así, que el investigador comprometido en el proceso investigativo.

Apoyado en Martínez, (1999) la posición metodológica consistió en escuchar detalladamente y en describir sucintamente cada uno de ellos y elaborar categorías y subcategorías representativamente de esas experiencias vivenciales" (pág. 69). Ciertamente para darles la palabra a los actores sociales sobre el significado atribuido a la supervisión educativa a través de sus experiencias vividas, permitiendo comprender esos estados experimentados, tratando de algún modo aproximarme a su realidad.

\section{Reflexiones finales}

A través de los planteamientos expuestos, la necesidad de supervisar ha estado siempre en las tareas fundamentales que desempeña el hombre para el desarrollo de los pueblos, que ésta a su vez siempre la ha normado un 
conjunto de preceptos que emiten los gobiernos, y al ser un proceso abierto, dinámico y complejo; necesita de muchas personas con roles definidos, que cumplan a cabalidad con la estructura organizativa que el gobierno de turno haya conformado según las necesidades educativas que existan en un momento puntual de la historia.

Asimismo es posible asegurar que la supervisión pueda y deba avalar la calidad educativa, conociendo las normativas vigentes, la realidad del estado docente, el desarrollo curricular, el control y seguimiento de los proyectos de aprendizaje estén basado en las necesidades de los educandos, asesorar a los gerentes educativos y docentes de aula, ya que la Supervisión como proceso indispensable dentro del contexto, por tener la tarea de conducción, orientación, asesoramiento, control y evaluación impulsan al proceso desde una óptica de ubicar la actualidad y el verdadero desarrollo de las acciones y responsabilidades para contribuir a la calidad de los resultados educativos conscientes, deliberados, participativos y permanente que se implementan en la estructura cuyo papel fundamental es el determinar situaciones a fin de demandar el cambio requerido para rectificar en aras de una mayor eficacia de la institución escolar; es decir, así se podría decir que la supervisión garantiza la calidad educativa en la educación primaria.

En nuestro país queda mucho trabajo por hacer para mejorar los resultados de las supervisiones y tomar acciones para los correctivos necesarios. Pero, para eso estamos nosotros. Para mejorar constantemente el proceso de Supervisión a través de nuevos paradigmas y desde nuestras propias experiencias podremos cambiar y guiar a las nuevas generaciones de maestros a ser excelentes y con alto sentido de pertinencia en su misión de educar para formar ciudadanos con una conciencia que les lleve a comprender, confrontar y verificar su realidad por sí mismos, que asimilen desde su entorno, para que sean copartícipes, protagónicos y corresponsables de su acción en la escuela. 


\section{Referencias}

Cano, E. (1998). Evaluación de la calidad educativa. Madrid: La Muralla.

Casanova, A. (2004). Evaluación y calidad de centros educativos. Madrid: La Muralla.

Ministerio de Educación y Deporte (2006). Escuelas Primarias Bolivarianas. Caracas, Venezuela.

English, F., y Hill, J. (1995). Calidad total en educación. Transformación de las escuelas en sitios de aprendizaje. Aplicación de las ideas de Deming a la enseñanza. México: Editorial Edamex.

Gil, A. (1855). De la instrucción pública en España. Tomo I. Madrid, Imprenta del Colegio de Sordomudos.

Ministerio del Poder Popular para La Educación (2013). "La Supervisión garantiza la calidad de la educación Venezolana". Caracas, Venezuela.

Ortega, N. (2014). La Revolución Bolivariana, la calidad de la educación y Luis Bonilla. [Documento en línea]. Recuperado de:

http://www.aporrea.org/educacion/a197330.html

Soler, E. (coord.) (1993). Fundamentos de supervisión educativa. Madrid: La Muralla. 
Jhoel Ruber Mota Fonseca

e-mail: jhoelmota@hotmail.com

Nació en San Felipe, estado Yaracuy. Cursa estudio de Doctorado en Ciencias de la Educación en la Universidad Fermin Toro en Cabudare estado Lara, Magister Scientiarum en Gerencia y Liderazgo en Educación en la Universidad Fermin Toro Cabudare estado Lara (2011). Profesor en Educación Integral de la Universidad Pedagógica Experimental Libertador- Instituto de Mejoramiento Profesional del Magisterio extensión Yaracuy (2005).

Actualmente se desempeña como Docente de Aula en la Escuela Primaria Bolivariana "Republica de Nicaragua" San Felipe estado Yaracuy desde Octubre de 2008 hasta la actualidad. Se desempeñó como Miembro del Equipo Técnico Zonal Fundación Samuel Robinson cumpliendo funciones de: Coordinador Metodológico Regional desde 2005 hasta Agosto 2008. fue facilitador durante año y medio en el Programa Nacional de Formación de Educadores de la Universidad Bolivariana de Venezuela.

A participado en más de quince jornadas de formación en áreas relacionadas a Investigación y Educación, también ha realizado seis ponencias y participado en dos Congresos Internacionales en el campo Educativo e investigativo Desde el 2012 hasta la actualidad se desempeña como Facilitador, Tutor y Jurado en el Programa de Maestría en Gerencia y Liderazgo en Educación y Docencia Universitaria (UFT) enlace San Felipe-Yaracuy.

El contenido de este manuscrito se difunde bajo una Licencia de Creative Commons ReconocimientoNoComercial-Compartirlgual 4.0 Internacional 